\title{
PREVALÊNCIA DE LESÕES POR FRICÇÃO EM IDOSOS COM CÂNCER E FATORES ASSOCIADOS
}

\author{
Chrystiany Plácido de Brito Vieira ${ }^{1}$ \\ Thalita Alves Teixeira ${ }^{1}$ \\ Telma Maria Evangelista de Araújo ${ }^{1}$ \\ Fernando José Guedes da Silva Júnior ${ }^{1}$ \\ Francisca Tereza de Galiza ${ }^{1}$ \\ Átila Sâmia Oliveira Rodrigues ${ }^{2}$
}

\author{
https://orcid.org/0000-0002-3429-3329 \\ https://orcid.org/0000-0002-3866-6989 \\ https://orcid.org/0000-0001-5628-9577 \\ https://orcid.org/0000-0001-5731-632X \\ https://orcid.org/0000-0001-5217-7180 \\ https://orcid.org/0000-0002-8091-5491
}

Objetivo: analisar a prevalência de lesões por fricção em idosos com câncer e fatores associados. Método: estudo transversal e analítico, realizado com 50 idosos, em serviço de Oncologia de um hospital escola, em Teresina-Piaui, Brasil. Utilizaram-se de entrevista, exame físico e prontuário para coleta dos dados, de fevereiro a abril de 2018. Realizaram-se análise descritiva, aplicação do teste Mann-Whitney para observar diferença das médias e da prova exata de Fischer para verificar associação entre desfecho e variáveis independentes. Resultados: média de idade 69,36 anos, maioria do sexo masculino (52,0\%), sem escolaridade (38,0\%), com comorbidades (74,0\%), em uso de anticoagulante (56\%) e corticoide (76\%), dependentes (76\%) e com déficit cognitivo (32\%). A prevalência de lesões foi $18 \%$, com localização predominante em membros superiores (64,6\%) e de classificação $2 b$ ( $50 \%$ ). Os fatores associados foram hematomas nas extremidades ( $p$-valor=0,003), uso de curativo adesivo ( $p$-valor=0,044) e presença de comorbidades ( $p$-valor=0,050). Conclusão: prevalência de lesões por fricção elevada, associada a fatores clínicos. Sugerem-se outras investigações para viabilizar ações preventivas mais eficazes e definir a epidemiologia dessas lesões, uma vez que são subnotificadas em serviços hospitalares de oncologia.

Descritores: Ferimentos e Lesões; Fricção; Idoso; Serviço Hospitalar de Oncologia; Enfermagem Oncológica.

\section{SKIN TEAR PREVALENCE AND ASSOCIATED FACTORS AMONG ELDERLY PEOPLE WITH CANCER}

Objective: To analyze the skin tear prevalence and associated factors in elderly people. Method: It was a cross-sectional and analytical study conducted with 50 elderly patients in the Oncology department of a school hospital, in Teresina-Piaui, Brazil. Interviews, physical exams and medical records were used for data collection from February to April 2018. Descriptive analysis was performed, with the Mann-Whitney test to observe difference in means and Fisher's exact test to verify the association between outcome and independent variables. Results: Mean age of 69.36 years, mostly male (52.0\%), without education (38.0\%), with comorbidities (74.0\%), anticoagulant (56\%) and corticoid (76\%) use, dependent (76\%), and cognitive deficit (32\%). There was an $18 \%$ prevalence of injuries, mostly in upper limbs (64.6\%) and Category $2 b$ (50\%). Associated factors were hematoma in the extremities ( $p$-value=0.003), use of adhesive dressing ( $p$-value=0.044), and presence of comorbidities ( $p$-value=0.050). Conclusion: There was a high skin tear prevalence associated with clinical factors. Further investigations are recommended to enable more effective preventive actions and to define the epidemiology of these lesions, since they are underreported in hospital oncology services.

Descriptors: Wounds and Injuries. Friction. Aged. Oncology Service, Hospital. Nursing.

\section{PREVALENCIA DE LESIONES POR FRICCIÓN EN ANCIANOS CON CÁNCER Y FACTORES ASOCIADOS}

Objetivo: analizar la prevalencia de lesiones por fricción y factores asociados en ancianos. Método: estudio transversal y analítico, realizado con 50 ancianos, en servicio de Oncología de hospital escolar, en Teresina-Piauí, Brasil. Se utilizaron entrevistas, examen físico y prontuario para recolección de datos, de febrero a abril de 2018. Se realizaron análisis descriptivos, aplicación del test Mann-Whitney para observar diferencia de las medias y exacto de Fischer para verificar la asociación entre desenlace y variables independientes. Resultados: la media de edad fue 69,36, la mayoría del sexo masculino (52\%), sin escolaridad (38,0\%), con comorbilidades (74,0\%), en uso de anticoagulante (56\%) y corticoide (76\%), dependientes (76\%) y con déficit cognitivo (32\%). La prevalencia de lesiones fue de $18 \%$, con localización predominante en miembros superiores (64,6\%) y de clasificación $2 \mathrm{~b}$ (50\%). Los factores asociados fueron hematomas en las extremidades ( $p$-valor=0,003), uso de curativo adhesivo ( $p$-valor=0,044) y presencia de comorbilidades ( $p$-valor=0,050). Conclusión: la prevalencia de lesiones por alta fricción estuvo asociada con factores clínicos. Se recomiendan otras investigaciones para permitir acciones preventivas más efectivas y definir la epidemiología de estas lesiones, ya que son subreportadas en servicios hospitalarios de oncología.

Descriptores: Lesiones y lesiones; Fricción; Anciano; Servicio Hospitalario de Oncología; Enfermería Oncológica.

'Universidade Federal do Piaui, PI, Brasil.

2Universidade de Fortaleza, CE, Brasil.

Autor correspondente: Chrystiany Plácido de Brito Vieira - Email: chrystianyplacido@yahoo.com

Recebido: 26/02/2020 - Aceito: 28/07/2020 


\section{INTRODUÇÃO}

Lesão por Fricção (LF) é considerada ferida traumática que ocorre, principalmente, nas extremidades de idosos, resultante de fricção ou combinação de fricção e cisalhamento, acarretando separação da epiderme e derme e/ou estruturas subjacentes ${ }^{1}$. Decorrem, basicamente, da fragilidade cutânea. Estas lesões são dolorosas e acometem, sobretudo, pacientes com extremos de idade, em cuidados críticos, com doenças crônicas e que necessitam de auxílio para atividades de vida diária².

Alguns pacientes que apresentam maior predisposição à ocorrência das LF, entre os quais estão os idosos, associada às alterações próprias do processo de envelhecer e às condições como mobilidade prejudicada, nutrição comprometida, necessidade de auxílio para atividades de vida diária e comorbidades, como o câncer ${ }^{3}$.

Com o envelhecimento, é natural a fragilidade, a redução da elasticidade e espessura das camadas da pele, o déficit hidroeletrolítico, que favorece o ressecamento e a descamação da pele, tornando-a mais friável e susceptível a lesões, as quais se potencializam após 75 anos de idade. ${ }^{4,5}$ No contexto da doença neoplásica, idosos apresentam maior incidência de alterações cutâneas e, por isso, é maior o risco de desenvolvimento de LF, devido ao tratamento oncológico e à progressão da doença².

Estudo aponta prevalência das LF variando de 3,3\% a $22 \%$, normalmente, associada à idade avançada e à dependência para atividades básicas de vida diária6. No Brasil, estudo realizado em 2010, com pacientes hospitalizados com câncer, encontrou prevalência de 3,3\%, sendo $60 \%$ da amostra acima de 60 anos de idade 4 .

As LF passam, muitas vezes, despercebidas, visto que são feridas traumáticas rasas. Juntam-se a essa problemática o fato de a epidemiologia das LF não está bem definida na literatura internacional e além da escassez de estudos no cenário nacional que avaliem a prevalência e os fatores associados a essas lesões ${ }^{6}$. Assim, faz-se necessária a construção de novos conhecimentos na área, a princípio, por meio de estudos que determinem prevalência desse tipo de lesão na população idosa, especialmente, com câncer, haja vista que a literatura descreve maiores riscos neste grupo específico.

Acredita-se que os resultados deste estudo ofereçam subsídios epidemiológicos desse tipo de ferida e dos fatores associados em idosos com câncer, o que poderá direcionar práticas de prevenção e tratamento em serviços hospitalares de oncologia e, ainda, servir de indicador de qualidade para prática assistencial da enfermagem oncológica.
Portanto, objetivou-se analisar a prevalência de lesões por fricção em idosos com câncer e os fatores associados.

\section{MÉTODO}

\section{Tipo de Estudo}

Estudo transversal e analítico, com amostragem por conveniência, desenvolvido em serviço de oncologia para adultos de hospital escola, em Teresina, Piauí, Brasil, com 16 leitos para internação e 12 para quimioterapia ambulatorial.

\section{Participantes da pesquisa}

A população do estudo foi constituída pelo universo dos casos de idosos com câncer $(n=50)$, atendidos no serviço de oncologia pesquisado, no período do estudo (fevereiro a abril de 2018). A seleção da amostra foi do tipo acidental, a qual se formou à medida que os idosos eram atendidos no setor. Incluíram-se, assim, os idosos que atenderam aos critérios: idade igual ou superior a 60 anos, estar internado nos leitos de enfermaria, no período da coleta dos dados, independentemente do tempo de internação, ou estar realizando quimioterapia ambulatorial.

\section{Coleta de dados}

$O$ instrumento utilizado na pesquisa constou de variáveis evidenciadas pela literatura como primordiais para identificar a associação entre a prevalência da LF com a pessoa idosa, sendo estas: sociodemográficas (idade, sexo, escolaridade, estado civil, aposentadoria e renda familiar); relacionadas à internação (local de atendimento, data da internação/atendimento, permanência hospitalar); clínicas (diagnóstico, peso, altura, Índice de Massa Corpórea-IMC, estado nutricional, comorbidades, quimioterapia, tabagismo, medicamentos de uso contínuo, aplicação de anticoagulante e corticoide, utilização de cateteres/drenos/sondas/curativos adesivos, condições atuais da pele, história prévia de queda e de LF, mobilidade, marcha, acuidade visual, Miniexame do Estado Mental-MEEM, Katz e presença ou não de lesão por fricção); e sobre LF (quantidade, localização, tempo de evolução dos ferimentos, infecção, retalho de pele e classificação).

A coleta de dados ocorreu por meio de entrevista, consulta ao prontuário e exame físico da pele do idoso, sendo realizada pelos pesquisadores, que receberam previamente treinamento com estomaterapeuta sobre reconhecimento e classificação da LF. Inicialmente, os idosos foram submetidos ao MEEM, com vistas a avaliar a capacidade cognitiva para responder à entrevista, e, caso acima do ponto de corte diferenciado, de acordo com o nível de escolaridade ${ }^{7}$, assinavam o Termo de Consentimento Livre e Esclarecido 
(TCLE). Caso apresentassem cognição comprometida, a entrevista era realizada com o responsável e o mesmo assinava o TCLE. Nesse momento, coletaram-se dados sociodemográficos e clínicos e, quando necessário, o formulário era complementado com informações obtidas dos prontuários (dados sobre as condições clínicas).

Durante a entrevista, era aplicada a Escala de Katz ${ }^{8}$ para avaliação da independência funcional para realização das Atividades Básicas de Vida Diária (ABVD). Os dados sobre peso e estatura foram extraídos da ficha de avaliação nutricional da instituição presente nos prontuários. Na sequência, realizou-se o exame físico da pele do idoso, no sentido cefalocaudal, para avaliação das condições da pele e identificação de LF. As lesões foram classificadas segundo o Sistema de Classificação STAR - Lesão por Fricção, nas categorias la, 1b, 2a, 2b e $3^{1}$. Destaca-se que cada idoso foi avaliado apenas uma vez.

\section{Procedimentos de análise dos dados}

Os dados foram submetidos ao processamento estatístico no software Statistical Package for the Social Science (SPSS), versão 22.0. Para caracterizar a amostra, realizaram-se estatísticas descritivas, por meio de distribuição de frequências e medidas de posição. O teste de Kolmogorou-Smirnou foi aplicado nas variáveis numéricas contínuas para verificação do pressuposto de normalidade, sendo encontrado padrão de distribuição não normal. Assim, para observar a diferença entre as médias das variáveis: idade (anos), renda familiar (reais), tempo de internação (dias), IMC $\left(\mathrm{kg} / \mathrm{m}^{2}\right)$, quantidade de medicamentos, tempo de uso de anticoagulante (dias), tempo de uso de corticoide (dias), escore do MEEM e da KATZ, com a ocorrência de lesão por fricção, aplicou-se o teste não paramétrico de Mann-Whitney. Para verificar associação entre variáveis qualitativas, utilizou-se do teste exato de Fisher. Adotou-se o nível de significância de 0,05.

\section{Aspectos éticos}

O estudo respeitou as exigências formais contidas nas normas nacionais e internacionais regulamentadoras de pesquisas. O projeto foi aprovado pelo Comitê de Ética em Pesquisa da Universidade Federal Piauí, conforme Parecer $n$ 으 2.085.462.

\section{RESULTADOS}

$\mathrm{Na}$ caracterização sociodemográfica, houve predominância da faixa etária de 60 a 79 anos de idade (86,0\%), com média de idade de 69,36, sexo masculino (52,0\%), casados $(60,0 \%)$, sem escolaridade (38,0\%), aposentados $(92,0 \%)$, sendo que $16,0 \%$ tinham renda familiar de até um salário mínimo (Tabela 1).

Tabela 1 - Características sociodemográficas dos idosos participantes $(n=50)$. Teresina, Piauí, 2018

\begin{tabular}{|c|c|c|c|c|}
\hline Variáveis & Mínima & Máxima & Média & $n(\%)$ \\
\hline Idade (em anos) & 60 & 96 & 69,36 & \\
\hline \multicolumn{4}{|l|}{$60-79$} & $43(86,0)$ \\
\hline \multicolumn{4}{|l|}{$\geq 80$} & $7(14,0)$ \\
\hline \multicolumn{5}{|l|}{ Sexo } \\
\hline \multicolumn{4}{|l|}{ Masculino } & $26(52,0)$ \\
\hline \multicolumn{4}{|l|}{ Feminino } & $24(48,0)$ \\
\hline \multicolumn{5}{|l|}{ Estado civil } \\
\hline \multicolumn{4}{|l|}{ Casado } & $30(60,0)$ \\
\hline \multicolumn{4}{|l|}{ Solteiro } & $6(12,0)$ \\
\hline \multicolumn{4}{|l|}{ Viúvo } & $9(18,0)$ \\
\hline \multicolumn{4}{|l|}{ Estável } & $5(10,0)$ \\
\hline $\begin{array}{l}\text { Escolaridade } \\
\text { (anos) }\end{array}$ & 0 & 11 & 3,34 & \\
\hline \multicolumn{4}{|l|}{ Sem escolaridade } & $19(38,0)$ \\
\hline \multicolumn{4}{|l|}{$\begin{array}{l}1-4 \text { anos } \\
5-10 \text { anos } \\
10 \text { ou mais }\end{array}$} & $\begin{array}{l}16(32,0) \\
13(26,0) \\
02(4,0)\end{array}$ \\
\hline \multicolumn{5}{|l|}{ Aposentadoria } \\
\hline \multicolumn{4}{|l|}{ Não } & $4(8,0)$ \\
\hline \multicolumn{4}{|l|}{ Sim } & $46(92,0)$ \\
\hline $\begin{array}{l}\text { Renda familiar } \\
\text { (SM reais) }\end{array}$ & 370 & 3000 & 1344,38 & \\
\hline \multicolumn{4}{|l|}{$\leq 1$} & $8(16)$ \\
\hline \multicolumn{4}{|l|}{$>1$} & $42(84)$ \\
\hline
\end{tabular}

Fonte: dados da pesquisa

Legenda: SM=Salário mínimo (R\$954,00)

Em relação à caracterização clínica dos idosos, verificou-se que $88,0 \%$ eram hospitalizados na enfermaria para tratamento clínico, com tempo médio de internação de 13,58 dias. Quanto à avaliação nutricional, 52,0\% eram de peso normal, seguido de $34,0 \%$ com sobrepeso, $10,0 \%$ baixo peso e $4,0 \%$ obesos. Dentre os entrevistados, $74,0 \%$ presentavam alguma comorbidade, com destaque para diabetes $(22,0 \%)$ e hipertensão $(60,0 \%)$.

Entre os entrevistados, 32,0\% estavam em quimioterapia, 70,0\% em uso de medicação contínua, 56,0\% usavam anticoagulante e $76,0 \%$ corticoide. No tocante à avaliação 
da pele, 64,0\% não usavam curativo adesivo, 28,0\% apresentavam equimoses, $16,0 \%$ hematomas nas extremidades, principalmente membros superiores, $22,0 \%$ tinham púrpura senil, $72,0 \%$ pele seca e descamativa e $20,0 \%$ edema em membros.

Verificou-se, ainda, que 40,0\% apresentavam marcha vacilante, $20,0 \%$ eram acamados, 32,0\% tinham comprometimento cognitivo, 34,0\% tinham acuidade visual prejudicada, assim 76,0\% eram dependentes para atividades de vida diária e 34,0\% apresentavam histórico de queda.

Constatou-se prevalência de LF de 18,0\% (IC95\%17,8418,16). Analisaram-se 14 lesões, média de 1,6 lesões por idoso. Quanto à localização, as regiões que predominaram foram MMSS (64,6\%) e tórax anterior (14,2\%). Nenhuma das lesões exibia sinais de infecção, mas quatro (28,5\%) apresentavam retalho viável, por isso metade obteve classificação 2 b (50,0\%) (Tabela 2$)$

Tabela 2 - Caracterização das lesões apresentadas (n=14). Teresina, Piauí, 2018.

\begin{tabular}{|l|l|}
\hline Variáveis & $\mathrm{n}(\%)$ \\
\hline Quantidade de LF por idoso $(\mathrm{n}=\mathbf{9})$ & \\
\hline 1 & $5(55,5)$ \\
\hline 2 & $3(33,3)$ \\
\hline 3 & $1(11,2)$ \\
\hline Localizações $(\mathrm{n}=14)$ & \\
\hline Tórax anterior & $2(14,2)$ \\
\hline Lombar & $1(7,1)$ \\
\hline Sacral & $1(7,1)$ \\
\hline MMSS & $9(64,6)$ \\
\hline MMII & $1(7,1)$ \\
\hline Infecção (n=14) & \\
\hline Não & $14(100)$ \\
\hline Sim & $0(0)$ \\
\hline Retalho de pele $(\mathbf{n}=14)$ & \\
\hline Viável & $4(28,5)$ \\
\hline Hematoma & $10(71,5)$ \\
\hline Classificação $(\mathbf{n}=14)$ & \\
\hline la & $3(21,5)$ \\
\hline lb & $4(28,5)$ \\
\hline 2b & $7(50,0)$ \\
\hline
\end{tabular}

Fonte: dados da pesquisa

Acerca da associação das LF com as variáveis sociodemográficas, observou-se que nenhuma variável apresentou associação estatisticamente significativa. Entre as variáveis clínicas, verificou-se associação entre a ocorrência de LF com presença de comorbidades $(p=0,050)$, uso de curativo adesivo $(p=0,044)$ e hematomas nas extremidades $(p=0,003)$ (Tabela 3).

Tabela 3 - Associação das variáveis sociodemográficas e clínicas com a ocorrência de LF (n=50). Teresina, Piauí, 2018.

\begin{tabular}{|l|l|l|l|}
\hline \multirow{2}{*}{ Variáveis } & \multicolumn{2}{|l|}{ Ocorrência de LF } & \\
\cline { 2 - 3 } & $\begin{array}{l}\text { Não } \\
\mathrm{n}(\%)\end{array}$ & $\begin{array}{l}\text { Sim } \\
\mathrm{n}(\%)\end{array}$ & p-valor* \\
\hline Sexo & \multicolumn{2}{|c|}{0,275} \\
\hline $\begin{array}{l}\text { Masculino } \\
\text { Feminino }\end{array}$ & $\begin{array}{l}\text { 20(48,7) } \\
\text { 21(51,2) }\end{array}$ & $\begin{array}{l}6(66,6) \\
3(33,4)\end{array}$ \\
\hline Idade (em anos) & & & 0,370 \\
$60-79$ & $36(87,8)$ & $7(77,7)$ & \\
$80-96$ & $5(12,2)$ & $2(22,3)$ & \\
\hline
\end{tabular}

Situação conjugal 0,169 Com companheiro $\quad 27(65,8) \quad 8(88,8)$ Sem companheiro $\quad$ 14(34,2) $\quad$ l(11,2)

$\begin{array}{cll}\text { Escolaridade } & & \\ \text { Sem } & 16(39,1) & 3(33,4) \\ \text { Com } & 25(60,9) & 6(66,6)\end{array}$

\begin{tabular}{|c|c|c|c|}
\hline Renda (SM) & & & 0,445 \\
\hline $\begin{array}{l}\geq 1 \\
>1\end{array}$ & $\begin{array}{l}6(14,6) \\
35(85,3)\end{array}$ & $\begin{array}{l}2(22,3) \\
7(77,7)\end{array}$ & \\
\hline Aposentadoria & & & 0,440 \\
\hline $\begin{array}{l}\text { Não } \\
\text { Sim }\end{array}$ & $\begin{array}{l}4(9,7) \\
37(90,2)\end{array}$ & $\begin{array}{l}0(0) \\
9(100)\end{array}$ & \\
\hline
\end{tabular}

\begin{tabular}{|c|c|c|c|}
\hline Local & & & 0,293 \\
\hline $\begin{array}{l}\text { Enfermaria } \\
\text { Ambulatório OT }\end{array}$ & $\begin{array}{l}37(90,2) \\
4(9,7)\end{array}$ & $\begin{array}{l}7(77,7) \\
2(22,3)\end{array}$ & \\
\hline Diagnóstico & & & 0,283 \\
\hline $\begin{array}{l}\text { Clínico } \\
\text { Cirúrgico }\end{array}$ & $\begin{array}{l}35(85,3) \\
6(14,6)\end{array}$ & $\begin{array}{l}9(100) \\
0(0)\end{array}$ & \\
\hline Comorbidades & & & 0,050 \\
\hline Não & $13(31,7)$ & $\mathrm{O}(0)$ & \\
\hline
\end{tabular}

$\begin{array}{lll}28(68,3) & 9(100) & \\ \text { Quimioterapia } & & 0,103\end{array}$

\begin{tabular}{|llll|}
\hline Não & $30(73,2)$ & $4(44,5)$ & \\
\hline Sim & $11(26,8)$ & $5(55,5)$ & \\
\hline Outros tratamentos & & & 0,517 \\
\hline Não & $34(82,9)$ & $7(77,7)$ & \\
Sim & $7(17,1)$ & $2(22,3)$ & \\
\hline Medicação de uso continuo & & & 0,549 \\
\hline Não & $12(29,2)$ & $3(33,4)$ & \\
Sim & $29(70,8)$ & $6(66,6)$ & \\
\hline Uso de anticoagulantes & & & 0,317 \\
\hline Não & $19(46,3)$ & $3(33,4)$ & \\
Sim & $22(53,6)$ & $6(66,6)$ & \\
\hline Uso de corticoide & & & 0,368 \\
\hline Não & $32(78,1)$ & $6(66,6)$ & \\
Sim & $9(21,9)$ & $3(33,4)$ & \\
\hline Uso de drenos ou cateteres & & & 0,510 \\
\hline Não & $12(29,2)$ & $2(22,3)$ & \\
Sim & $29(70,8)$ & $7(77,7)$ & \\
\hline Uso de sondas & & & 0,416 \\
\hline Não & & \\
Sim & $32(78,1)$ & $8(88,8)$ & \\
\hline
\end{tabular}

Enferm. Foco 2020; 11 (4) 58-65 | 61 


\begin{tabular}{|c|c|c|c|}
\hline Uso de curativos adesivos & & & 0,044 \\
\hline $\begin{array}{l}\text { Não } \\
\text { Sim }\end{array}$ & $\begin{array}{l}29(70,8) \\
12(29,2)\end{array}$ & $\begin{array}{l}3(33,4) \\
6(66,6)\end{array}$ & \\
\hline Equimose & & & 0,207 \\
\hline $\begin{array}{l}\text { Não } \\
\text { Sim }\end{array}$ & $\begin{array}{l}31(45,6) \\
10(24,4)\end{array}$ & $\begin{array}{l}5(55,5) \\
4(44,5)\end{array}$ & \\
\hline $\begin{array}{l}\text { Hematomas nas extremi- } \\
\text { dades }\end{array}$ & & & 0,003 \\
\hline $\begin{array}{l}\text { Não } \\
\text { Sim }\end{array}$ & $\begin{array}{l}38(92,6) \\
3(7,4)\end{array}$ & $\begin{array}{l}4(44,5) \\
5(55,5)\end{array}$ & \\
\hline Purpura senil & & & 0,308 \\
\hline $\begin{array}{l}\text { Não } \\
\text { Sim }\end{array}$ & $\begin{array}{l}33(80,4) \\
8(19,6)\end{array}$ & $\begin{array}{l}6(66,6) \\
3(33,4)\end{array}$ & \\
\hline Pele seca e descamativa & & & 0,207 \\
\hline $\begin{array}{l}\text { Não } \\
\text { Sim }\end{array}$ & $\begin{array}{l}10(24,4) \\
31(45,6)\end{array}$ & $\begin{array}{l}4(44,5) \\
5(55,5)\end{array}$ & \\
\hline Edema em extremidades & & & 0,249 \\
\hline $\begin{array}{l}\text { Não } \\
\text { Sim }\end{array}$ & $\begin{array}{l}34(82,9) \\
7(17,1)\end{array}$ & $\begin{array}{l}4(44,5) \\
5(55,5)\end{array}$ & \\
\hline Acuidade visual & & & 0,358 \\
\hline $\begin{array}{l}\text { Preservada } \\
\text { Comprometida }\end{array}$ & $\begin{array}{l}28(68,3) \\
13(31,7)\end{array}$ & $\begin{array}{l}5(55,5) \\
4(44,5)\end{array}$ & \\
\hline Total & $41(100)$ & $9(100)$ & \\
\hline
\end{tabular}

Fonte: dados da pesquisa

Legenda: *Teste exato de Fisher

Conforme Tabela 4, houve diferença estatisticamente significativa na média da renda familiar $(p=0,040)$ e do escore do MEEM ( $p=0,018)$, quando comparados idosos com e sem LF.

Tabela 4 - Comparação de médias das variáveis sociodemográficas e clínicas com a ocorrência de LF ( $n=50)$. Teresina, Piauí, 2018.

\begin{tabular}{|l|l|l|l|}
\hline \multirow{2}{*}{ Variáveis } & \multicolumn{2}{l}{ Ocorrência de LF } & \multirow{2}{*}{ p-valor* } \\
\cline { 2 - 3 } & $\begin{array}{l}\text { Não } \\
(\end{array}$ & $\begin{array}{l}\text { Sim } \\
()\end{array}$ & \\
\hline Idade (anos) & 68,80 & 71,88 & 0,855 \\
\hline Renda familiar (reais) & 1314,60 & 1480,00 & 0,040 \\
\hline Tempo de internação (dias) & 13,92 & 12,00 & 0,915 \\
\hline IMC & 23,66 & 22,03 & 0,891 \\
\hline $\begin{array}{l}\text { Quantidade de medicamen- } \\
\text { tos }\end{array}$ & 1,80 & 2,11 & 0,931 \\
\hline $\begin{array}{l}\text { Tempo de uso de anticoagu- } \\
\text { lante (dias) }\end{array}$ & 9,82 & 9,33 & 0,954 \\
\hline $\begin{array}{l}\text { Tempo de uso de corticoide } \\
\text { (dias) }\end{array}$ & 5,31 & 6,55 & 0,751 \\
\hline MEEM & 18,63 & 16,44 & 0,018 \\
\hline KATZ & 3,02 & 4,11 & 0,173 \\
\hline
\end{tabular}

Fonte: dados da pesquisa

Legenda: = Média; $\left(^{*}\right) \bigcirc \mathrm{p}$ valor foi obtido pelo teste Mann-Whitney.

\section{DISCUSSÃO}

Trata-se do primeiro estudo regional sobre a prevalência de LF em idosos com câncer. Apesar desta lesão ocorrer principalmente na idade avançada, devido à fragilidade da pele, não se encontraram estudos nacionais sobre associação da epidemiologia das LF a esta população.

$\mathrm{O}$ processo de envelhecimento populacional reflete a elevada ocorrência de doenças crônicas não transmissiveis associadas à maior fragilidade do corpo, entre estas, cita-se o câncer, doença tipicamente concentrada nas faixas etárias mais avançadas 9 . Essa realidade demanda planejamento, organização e avaliação do cuidado de enfermagem, considerando as respostas diferenciadas ao tratamento, bem como as limitações específicas dessa faixa etária, relacionadas aos possíveis problemas sensoriais, de memória e locomoção ${ }^{10}$

Assim, destaca-se a importância de se averiguar a prevalência e os fatores associados à LF em idosos com câncer, pois apresentam condições clínicas resultantes da idade, doença e do tratamento que aumentam o risco da ocorrência dessas lesões ${ }^{2}$, trazendo implicações diretas na qualidade de vida desta parcela da população.

Destacou-se, no presente estudo, a faixa etária de 60 a 79 anos, como também apontado em outros estudos ${ }^{10-12}$. Os dados estão em consonância aos descritos pelo Instituto Nacional do Câncer (INCA), cuja tendência de aumento na incidência em faixas etárias a partir dos 60 anos de idade é mencionada ${ }^{13}$ em função das transições demográfica e epidemiológica globais que sinalizam impacto, cada vez maior, da carga de câncer nas próximas décadas.

A predominância do sexo masculino também é constatada pelas estimativas do INCA, que apontam discreto predomínio do sexo masculino, tanto na incidência (53\%) quanto na mortalidade $(57 \%)^{13}$. Possível explicação poderia ser encontrada no fato da não adesão de homens às medidas de atenção integral, fator contribuinte para que estes cuidem menos de si e se exponham mais às situações de risco ${ }^{14}$.

Em relação à situação conjugal, a maior parte dos idosos era casada, o que demonstrou possuir apoio familiar, importante fator frente ao cuidado e tratamento de tal patologia ${ }^{15}$. A família deve ser considerada por enfermeiros como aliada para implementação das medidas de cuidado.

No tocante à renda, constataram-se idosos com renda menor de um salário mínimo. Pessoas com níveis socioeconômicos mais baixos têm apresentado maior incidência de câncer em geral, maior proporção de diagnóstico tardio, maior dificuldade de acesso ao diagnóstico e tratamento adequado, pior prognóstico e menor so- 
brevida após o diagnóstico de câncer, maior risco de óbito por câncer em geral e por tipos de câncer potencialmente curáveis ${ }^{13}$.

A escolaridade baixa reflete o grau de conhecimento do paciente, o que, associado à baixa renda, constitui vulnerabilidade para muitos processos de saúde-doença, como nas neoplasias, pois reduz as ações de prevenção e as chances de acesso aos serviços de saúde, podendo resultar em diagnóstico tardio ${ }^{16}$

Quanto à caracterização clínica dos idosos, a maioria apresentava outras comorbidades além do câncer em tratamento. O acometimento das doenças crônicas com o avanço da idade é resultado esperado pelas características dessas doenças, associado à ocorrência simultânea de diferentes fatores de risco, ao uso contínuo e diversificado de medicamentos e às mudanças corporais e funcionais inerentes ao processo de envelhecimento, o que exige cuidado especial voltado a essa fase da vida ${ }^{13,16}$.

No que diz respeito à avaliação nutricional, a maioria possuía peso normal. O tratamento quimioterápico é um dos fatores que podem induzir ao aparecimento de infecções e contribuir para piora do estado nutricional. Outro fator é o tempo de permanência hospitalar que também é uma das causas de risco nutricional em pacientes oncológicos, considerando que, de forma geral, estes pacientes se mantêm internados por maior período ${ }^{17}$.

Quanto aos medicamentos, maioria utilizava medicação de uso contínuo, dentre eles corticoides e anticoagulantes. A polifarmácia e o uso prolongado de esteroides e anticoagulantes, associados à fragilidade da pele dos idosos, predispõem o aparecimento de LF ${ }^{18-19}$.

No que diz respeito à prevalência de LF, apesar de que na literatura não se encontraram trabalhos associando a ocorrência dessa lesão nos pacientes idosos em serviço hospitalar de oncologia, verificou-se elevada taxa de prevalência. Estudo realizado, no Brasil, com pacientes hospitalizados com câncer, levantou a prevalência de $3,3 \%{ }^{3}$, bem abaixo de 18,0\% da encontrada neste estudo, cuja explicação pode estar no fato de a população ser somente de pacientes idosos.

Acerca das características das lesões apresentadas, a categoria $2 b$ foi a mais prevalente, ou seja, todas apresentavam retalho, mas viável em apenas $28,5 \%$ das lesões analisadas. Este achado diverge de outros estudos, um nacional $^{3}$, em que prevaleceu as de categoria 3, e outro internacional, que predominou $1 b^{20}$. Destaca-se que não há uso de linguagem comum na identificação e classificação das LF em todos os ambientes de cuidados com a saúde, no entanto, a definição uniforme da LF pode auxiliar na padroni- zação do ensino clínico, prática, comunicação, planejamento de cuidados e pesquisa entre profissionais de saúde ${ }^{21}$

A localização mais frequente foi nos membros superiores, corroborando com outros estudos ${ }^{6,19}$ que apontam que essa região é comum em idosos dependentes, situação comprovada neste estudo, em que a maioria dos participantes era dependente para atividades de vida diária.

Com relação aos fatores de risco associados com LF, a literatura aponta idade avançada, dependência para atividades básicas de vida diárias, mobilidade, comportamento agitado, comprometimento cognitivo, espasticidade, uso de anticoagulantes, equimoses e história prévia de $L^{3,20}$, o que diverge dos dados apontados neste estudo, exceto comprometimento cognitivo, pois se verificou que a ocorrência de LF estava associada à menor média do MEEM.

No presente estudo, a ocorrência de LF esteve associada com as características clínicas: uso de curativo adesivo, hematomas nas extremidades e presença de comorbidades. O uso do curativo adesivo, especificamente a retirada do curativo adesivo, é considerada uma das causas extrínsecas para ocorrência de LF, por se tratar de um trauma ${ }^{21}$.

A presença de hematomas nas extremidades, identificada na maioria dos idosos avaliados, principalmente em membros superiores, localização mais prevalente das lesões, aumenta o risco de trauma, quando existe a necessidade de realização das atividades de vida diária, bem como de transferir o paciente. A redução da lubrificação natural da pele, associada ao envelhecimento, torna-os mais susceptiveis à fricção e ao cisalhamento que podem resultar em ferimentos e lesões ${ }^{22}$

A presença de comorbidades pode comprometer a saúde global e, em pacientes com câncer que estão mais susceptíveis ao comprometimento da saúde da pele, aumentam ainda mais as chances da ocorrência de LF. A neoplasia compromete os mecanismos homeostáticos do corpo, resultando na diminuição da perfusão da pele e das partes moles e em prejuízos nos processos metabólicos cutâneos, deixando a pele mais susceptivel a lesões. A ocorrência de outros fatores, além da própria idade avançada, como doenças crônicas, aumenta sobremaneira a fragilidade e vulnerabilidade dérmica ${ }^{3}$

Outra condição que eleva a vulnerabilidade de idosos com câncer à ocorrência de LF é o comprometimento cognitivo, uma vez que pode trazer incapacidade para cuidar de si e comportamento pouco colaborativo, situações comuns entre os pacientes oncológicos com LF, pois acarreta prejuízo da mobilidade física e aumenta a dependência para atividades básicas de vida diáriab.

Baixa renda é fator importante a ser considerado quan- 
to à vulnerabilidade de idosos com câncer, no entanto, constatou-se, neste estudo, na comparação de média, que os idosos com LF apresentavam média maior de renda. Embora maior renda e alta escolaridade exerçam fatores protetores para esses idosos, não os tornam totalmente isentos de desenvolverem lesões na pele, como as LF, haja vista que as modificações cutâneas e nos demais sistemas, decorrentes do envelhecimento ${ }^{4}$, aumentam o risco de lesões e complicações.

\section{Limitações do estudo}

As limitações do estudo estão relacionadas principalmente à casuística e amostra intencional, pois foi realizado em apenas uma instituição hospitalar especializada, o que dificultou também a realização de estatística mais robusta.

\section{Contribuições do estudo}

Este estudo pode contribuir para o melhor conhecimento da epidemiologia das LF em pacientes idosos com câncer. Logo, sugere-se a realização de estudos como este em outros serviços de oncologia para levantamento da problemática, com vistas a contribuir para ações preventivas mais adequadas pela enfermagem oncológica, com base em investigações bem delineadas.

\section{CONCLUSÃO}

Constatou-se prevalência de $18,0 \%$ de LF, o que permitiu concluir que estas constituem problema para pacientes idosos com câncer hospitalizados, estando associadas à maior média da renda familiar, à menor média do MEEM, ao uso de curativo adesivo, a hematomas nas extremidades e à presença de comorbidades. Trata-se de problema que existe em serviços hospitalares de oncologia e que precisa ser adequadamente abordado, para viabilizar ações preventivas mais eficazes e definir a epidemiologia dessas lesões, uma vez que são subnotificadas nos serviços.

\section{Contribuições dos autores:}

CPBV: concepção e desenho, análise e interpretação dos dados, redação do artigo, revisão crítica e revisão final; TAT: concepção e desenho, análise e interpretação dos dados, redação do artigo, revisão crítica e revisão final TMEA: concepção e desenho, análise e interpretação dos dados, redação do artigo, revisão crítica e revisão final; FJGSJ: análise e interpretação dos dados, redação do artigo, revisão crítica e revisão final; FTG: análise e interpretação dos dados, redação do artigo, revisão crítica e revisão final; ASOR: análise e interpretação dos dados, redação do artigo, revisão crítica e revisão final.

\section{REFERÊNCIAS}

1. Strazzieri-Pulido KC, Santos VLCG. Cultural adaptation and validation of STAR Skin Tear Classification System for Brazilians. Wound Stomy Continence Nurs J [Internet]. 2011; 38(3S):92. Available from: http://www.scielo.br/scielo.php?script=sci_nlinks\&pid =S1983-1447201400020014200017\&lng=en

2. Baranoski S, Leblanc K, Gloeckner M. CE: preventing, assessing, and managing skin tears: a clinical review. Am J Nurs [Internet]. 2016; 116(11):24-30. Available from: https:// doi.org/10.1097/01.NAJ.0000505581.01967.75

3. Amaral AFS, Pulido KCS, Santos VLCG. Prevalence of skin tears among hospitalized patients with cancer. Rev Esc Enferm USP [Internet]. 2012; 46(n. esp):44-50. Available from: http://dx.doi.org/10.1590/S0080-62342012000700007

4. Santos EI. Skin tear treatment and prevention by nurses: an integrative literature review. Rev Gaúcha Enferm [Internet]. 2014; 35(2):142-9. Available from: http://dx.doi. org/10.1590/1983-1447.2014.02.45178
5. Girondi JBR, Soldera D, Evaristo SM, Locks MOH, Amante LN, Vieira AS. Desbridamento de feridas em idosos na atenção primária em saúde. Enferm Foco [Internet]. 2019 10(5):20-5. Available from: http://revista.cofen.gov.br/index.php/enfermagem/article/view/2669

6. Strazzieri-Pulido KC, Peres GRP, Campanili TCGF, Santos VLCG. Skin tear prevalence and associated factors: a systematic review. Rev Esc Enferm USP [Internet]. 2015 49(4):674-80. Available from: http://dx.doi.org/10.1590/ S0080-623420150000400019

7. Bertolucci PHF, Brucki SMD, Campacci SR, Juliano Y. O mini-exame do estado mental em uma população geral. Impacto da escolaridade. Arq Neuropsiquiatr [Internet] 1994; 52(1):1-7. Available from: http://dx.doi.org/10.1590/ S0004-282X1994000100001

8. Lino VTS, Pereira SRM, Camacho LAB, Ribeiro Filho ST, Buksman S. Adaptação transcultural da Escala de Independência em Atividades de Vida Diária (Escala de 
Katz). Cad Saúde Pública [Internet]. 2008; 24(1):10312. Available from: http://dx.doi.org/10.1590/SO102$311 \times 2008000100010$

9. Matoso LML, Costa Oliveira LE, Oliveira KKD. Perfil Sociodemográfico dos portadores de neoplasias nos serviços de referência do Rio Grande do Norte. Rev Eletr Fainor [Internet]. 2016; 9(1). Available from: srv02.fainor. com.br/revista/index.php/memorias/article/download/487/278.

10. Silva JA, Hansel CG, Silva J. Qualidade de vida na perspectiva de idosos com câncer: implicações para enfermagem na atenção básica. Rev Enferm UERJ [Internet]. 2016; 24(3):e9621. Available from: https://doi. org/10.12957/reuerj.2016.9621

11. Santos CA, Ribeiro AQ, Rosa COB, Ribeiro RCL. Depressão, déficit cognitivo e fatores associados à desnutrição em idosos com câncer. Ciênc Saúde Coletiva [Internet]. 2015; 20(3):751-60. Available from: http://dx.doi. org/10.1590/1413-81232015203.06252014

12. Silva DVA, Carmo JR, Cruz MEA, Rodrigues CAO, Santana ET, Araújo DD. Caracterização clínica e epidemiológica de pacientes atendidos por um programa público de atenção domiciliar. Enferm Foco [Internet]. 2019; 10(3):112-8. Available from: http://revista.cofen.gov.br/ index.php/enfermagem/article/view/1905/572

13. Instituto Nacional de Câncer José Alencar Gomes da Silva (INCA). Coordenação de Prevenção e Vigilância. Estimativa 2018: incidência de câncer no Brasil. INCA: Rio de Janeiro; 2017

14. Fernandes AAC, Leandro FS, Oliveira GS, Araújo CLO Sexualidade em homens com câncer de próstata. Rev Eletr Enferm Vale do Paraiba [Internet]. 2015; 1(6). Available from: fatea.br/seer3/index.php/REENVAP/article/ download/57/46/

15. Lima AP, Lini EV, Giacomazzi RB, Dellani MP, Portella MR, Doring M. Prevalência e fatores associados à realização de exames de câncer de próstata em idosos: estudo de base populacional. Rev Bras Geriatr Gerontol [Internet]. 2018; 21(1):53-9. Available from: http://dx.doi. org/10.1590/1981-22562018021.170054

16. Haddad NC, Carvalho ACA, Novaes CO. Perfil sociodemográfico e de saúde de mulheres submetidas à cirurgia para câncer de mama. Rev Hospital Universitário Pedro Ernesto [Internet]. 2015. Available from: https:// doi.org/10.12957/rhupe.2015.17923

17. Coruja MK, Steemburgo T. Estado nutricional e tempo de internação de pacientes adultos hospitalizados com diferentes tipos de câncer. Braspen J [Internet]. 2017; 32(2):114-8. Available from: http://www.braspen.com.br/ home/wp-content/uploads/2017/08/03-AO-Estado-nutricional-e-tempo.pdf

18. Powell RJ, Hayward CJ, Snelgrove CL, Polverino K, Park L, Chauhan R et al. Pilot randomised controlled trial of protective socks against usual care to reduce skin tears in high risk people "STOPCUTS": study protocol. Pilot Feasibility Stud [Internet]. 2015; 1(1):12. Available from: http://dx.doi.org/10.1186/s40814-015-0005-3

19. Stephen-Haynes J, Callaghan R. The prevention, assessment and management of skin tears. Wounds UK [Internet]. 2017; 13(2):58-65. Available from: https:// www.wounds-uk.com/resources/details/prevention-assessment-and-management-skin-tears

20. Koyano Y, Nakagami G, Iizaka S, Minematsu T, Noguchi H, Tamai N, Mugita Y et al. Exploring the prevalence of skin tears and skin properties related to skin tears in elderly patients at a long term medical facility in Japan. Int Wound J [Internet]. 2014. Available from: http:// dx.doi.org/10.111l/iwj.12251

21. Chang YY, Carville K, Tay AC. The prevalence of skin tears in the acute care setting in Singapore. Int Wound J [Internet]. 2016; 13(5):977-83. Available from: http://dxdoi.org/10.1111/iwj.12572

22. Leblanc K, Baranoski S. Skin tears: state of the science: consensus statements for the prevention, prediction, assessment, and treatment of skin tears $\odot$. Adv Skin Wound Care [Internet]. 2011; 24(9):2-15. Available from: http://dx.doi.org/10.1097/01.ASW.0000405316. 99011.95 